\title{
Moment Stability of the Critical Case of PWM Feedback Systems with Stochastic Perturbations
}

\author{
Zhong Zhang and Lixia Ye \\ College of Mathematics and Statistics, Chongqing University, Chongqing 400044, China \\ Correspondence should be addressed to Zhong Zhang, zhanguicqu@gmail.com
}

Received 13 August 2012; Accepted 20 September 2012

Academic Editor: Chuandong Li

Copyright (C) 2012 Z. Zhang and L. Ye. This is an open access article distributed under the Creative Commons Attribution License, which permits unrestricted use, distribution, and reproduction in any medium, provided the original work is properly cited.

This paper further studies the moment stability of pulse-width-modulated (PWM) feedback system which is subjected to multiplicative and additive random disturbance modeled by the derivative of Wiener process. Different from the existing investigation, we focus on its critical case. The linear plant considered herein is assumed to be critically stable; that is, the plant has one and only one pole at the origin, and the rest of the poles are left half of complex plane. We establish several globally asymptotically stability criteria for such PWM feedback systems and then propose an algorithm to calculate the stability bound effectively. Furthermore, we present two numerical examples to show the effectiveness of the theoretical results.

\section{Introduction}

Pulse-width modulation has extensively been used in attitude control systems, adaptive control systems, signal processing, power control systems, modeling of neuron behavior, and the like (e.g., see [1-5]). In many areas, especially engineering applications, it is always operated in all kinds of accidental or continued disturbances. It is of prime importance whether or not keeping the scheduled operation or work of the state. Therefore, there has been a growing research interest on the stability analysis for PWM feedback systems, and a set of stability results have been established by a variety of methods [6-20]. By the method of positive kernels of integral operator, Halanar in [10] proposed two versions of direct Lyapunov and Lagrange stability results for PWM feedback system with Hurwitz stable plant. In $[12,13]$, the authors studied the stability properties of nonlinear PWM feedback systems.

There are, however, only a few results concerning the qualitative properties of PWM feedback system subjected to random disturbance. Gupta and Jury [14] developed a method 
of determining the mean square value of the output of a PWM system with Gaussian random input. Sun et al. [15] presented a set of theoretical results for mean square exponential stability, asymptotical mean square stability, and the $p$ th moment exponential stability by using spectrum technique. Besides, in [16], the authors gave some definitions on the $p$ th moment stable in mean and established several $p$ th moment globally stability criteria in mean. Recently, the authors in $[17,18]$ investigated the dynamical systems subjected to noise disturbance by linear matrix inequalities (LMIs) and control Lyapunov methods. In [20], the authors established new Lyapunov and Lagrange stability results for pulse-width-modulated (PWM) feedback systems subjected to random disturbance. The linear plant considered therein was assumed to be Hurwitz stable. An optimization procedure was also presented in [20], which is expected to improve the analysis performance significantly. And then they have shown that when the parameters of PWM are within a certain computable range, the PWM feedback system is stable.

To the best of the authors' knowledge, there are a few (if any) results for the stability analysis of the critical case of PWM systems with stochastic perturbations. In the present paper, we try to make the contribution on this issue. It is noted that the linear plant considered herein is critically stable; that is, the linear plant has one and only one pole at the origin, and the rest of the poles are left half of complex plane. Obviously, such systems are somewhat complex in comparison with most of the systems in literature. We will establish several Lyapunov and Lagrange criteria for the $p$ th moment uniform asymptotical stability in mean and then present an algorithm to compute the upper bound for the parameters of PWM. We will characterize the relationship between the parameters of PWM and the coefficients of state vectors of the feedback systems. It will be shown that when the random disturbance is sufficiently small, such PWM feedback system is the $p$ th moment globally asymptotically stable in mean provided that the upper bounds of parameters of pulse-width modulator are selected properly. We also demonstrate the effectiveness of our results by means of two numerical examples.

\section{Notations and Some Definitions}

We let $(\Omega, F, P)$ denote the underlying probability space for all the systems that will be considered, where $\Omega$ is the sample space, $F$ is the $\sigma$-algebra of subsets of the sample space, and $P$ is the probability measure. An $R^{n}$-valued random variable $x$ with domain $X$ is a measurable function from $\Omega$ to $X \subset R^{n}$. A family $\{x(\mathrm{t}), t \in I\}$ of $R^{n}$-valued random variables with domain $X$ defined on a probability space $(\Omega, F, P)$ is called a stochastic process with index set $I$ and state space $\left(X, F^{n}\right)$.

Definition 2.1. Let $(X, d)$ be a metric space, $X \subset R^{n}, A \subset X$, and let $T \subset R^{+}$. For any fixed $a \in A$ ( $a$ is called the initial state), $t_{0} \in T$, a stochastic process $\left\{x\left(t, \omega, a, t_{0}\right), t \in T_{a, t_{0}}\right\}$ with domain $X$ is called a stochastic motion if $x\left(t_{0}, \omega, a, t_{0}\right)=a$ for all $\omega \in \Omega$, where $T_{a, t_{0}}=\left[t_{0}, t_{1}\right) \cap T, t_{1}>t_{0}$, and $t_{1}$ is finite or infinite.

Definition 2.2. Let $S$ be a family of stochastic motions with domain $X$ given by

$$
S \subset\left\{x\left(\cdot, \cdot, a, t_{0}\right): x\left(t_{0}, \omega, a, t_{0}\right)=a, \omega \in \Omega, a \in A, t_{0} \in T\right\} .
$$

We call the four-tuple $\{T, X, A, S\}$ a stochastic dynamical system. 
Definition 2.3. Let $\{T, X, A, S\}$ be a stochastic dynamical system. A set $M \subset A$ is said to be invariant with respect to system $S$ (or short, $(S, M)$ is invariant) if $a \in M$ implies that

$$
P\left\{\omega: x\left(t, \omega, a, t_{0}\right) \in M \forall t \in T_{a, t_{0}}\right\}=1, \quad \forall t_{0} \in T \text {, and all } x\left(\cdot, \cdot, a, t_{0}\right) \in S .
$$

Definition 2.4. $x_{0} \in A$ is called an equilibrium point of a stochastic dynamical system $\{T, X, A, S\}$ if the set $\left\{x_{0}\right\}$ is invariant with respect to $S$.

Definition 2.5. $S$ is said to be the pth moment uniformly bounded if, for every $\eta>0$ and for every $t_{0} \in T$, there exists a $\beta=\beta(\eta)>0$ such that if $d\left(a, x_{0}\right)<\eta$, then for all $x\left(\cdot, \cdot, a, t_{0}\right) \in$ $S, E d\left(x\left(t, \omega, a, t_{0}\right), x_{0}\right)^{p}<\beta$ for all $t \in T_{a, t_{0}}$, where $t_{0}$ is a fixed point in $X$. $S$ is said to be the $p t h$ moment uniformly ultimately bounded in mean if there exists $B>0$ and if for every $\delta>0$ and for every $t_{0} \in T$ there exists a $\tau=\tau(\delta)$ such that for all $x\left(\cdot, \cdot, a, t_{0}\right) \in S, E d\left(x\left(t, \omega, a, t_{0}\right), x_{0}\right)^{p}<\beta$, for all $t>t_{0}+\tau$, whenever $d\left(a, x_{0}\right)<\delta$, where $x_{0}$ is a fixed point in $X$.

Definition 2.6. Let $\{T, X, A, S\}$ be a stochastic dynamical system, and let $d$ be the metric on $X$. A set $M \subset A$ is said to be the $p$ th moment stable in mean (i.e., $(S, M)$ is said to be the $p$ th moment stable in mean) if, for any $\varepsilon>0, t_{0} \in T$, there exists $\delta=\delta\left(t_{0}, \varepsilon\right)$ such that $E d\left(x\left(t, \omega, a, t_{0}\right), M\right)^{p}<\varepsilon$ for any process $x\left(\cdot, \cdot, a, t_{0}\right) \in S$, whenever $d(a, M)<\delta$, where $E$ denotes the expectation of a random process. If $\delta$ is independent of $t_{0},(S, M)$ is said to be the pth moment uniformly stable in mean. $(S, M)$ is said to be the pth moment uniformly asymptotically stable in mean if it is uniformly stable in the $p$ th mean and if, for any $\varepsilon>0$, there exists a $\delta=\delta(\varepsilon)>0$ and a $\tau=\tau(\varepsilon)$ such that for any process $x\left(\cdot, \cdot, a, t_{0}\right) \in S$, whenever $d(a, M)<\delta$ implies that $E d\left(x\left(t, \omega, a, t_{0}\right), M\right)^{p}<\varepsilon$ for all $t>t_{0}+\tau$.

We can similarly define the $p$ th moment asymptotic stability in mean, the $p$ th moment asymptotic stability in mean in the large, thepth moment uniform asymptotic stability in mean in the large, the $p$ th moment exponential stability in mean, and the $p$ th moment exponential stability in mean in the large. When $p=2$, we speak of various stability and bound concepts in the mean square.

\section{Stability Analysis of PWM Feedback System: The Critical Case}

The PWM feedback system to be considered in this paper is shown in Figure 1.

The pulse-width modulator is described by

$$
u(t)=m(e(t))= \begin{cases}M \operatorname{sgn}(e(k T)) & t \in\left[k T, k T+T_{k}\right), \\ 0 & t \in\left[k T+T_{k},(k+1) T\right),\end{cases}
$$

where $e(t)=r(t)-y(t)$ with $r(t)$ being the external input and $y(t)$ the system output, and, for $k=0,1,2, \ldots$, the pulse-width $T_{k}$ and the sign function sign are given, respectively, by

$$
T_{k}= \begin{cases}\beta|e(k T)|, & |e(k T)| \leq \frac{T}{\beta} \\ T, & |e(k T)|>\frac{T}{\beta}\end{cases}
$$




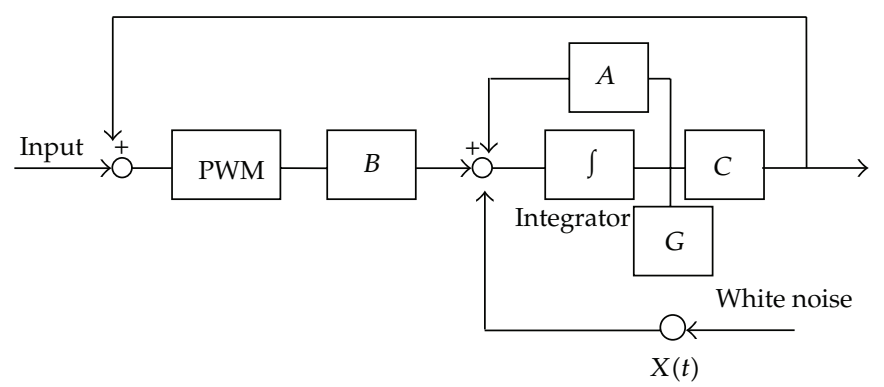

Figure 1: Block diagram of PWM feedback systems subjected to multiplicative disturbances [20].

$$
\operatorname{sgn}(\sigma)= \begin{cases}1 & \sigma>0 \\ 0 & \sigma=0 \\ -1 & \sigma<0\end{cases}
$$

The sampling period $T$, the amplitude of the pulse $M$, and $\beta$ are all assumed to be constants. And throughout this paper, we always assume that $r(t) \equiv 0$. Under these assumptions, the PWM feedback system with the output function can be described by

$$
\begin{gathered}
d x(t)=A x(t) d t+B u(t) d t+G x(t) d W_{t}, \\
y(t)=C x(t),
\end{gathered}
$$

where $x \in R^{n}, y \in R, u \in R$ is output of the pulse-width modulator, $A, B, C$, and $G$ are matrices of appropriate dimensions, and $W(t)$ is a scalar wiener process.

Furthermore, we assume that the linear plant is critically stable; that is, the matrix $A$ is stable with one and only one eigenvalue equal to zero. Without loss of generality, we assume that

$$
A=\left(\begin{array}{cc}
A_{1} & 0 \\
0 & 0
\end{array}\right), \quad B=\left(\begin{array}{c}
B_{1} \\
b
\end{array}\right), \quad C=\left[\begin{array}{ll}
C_{1} & 1
\end{array}\right], \quad G=\left(\begin{array}{cc}
G_{1} & 0 \\
0 & g
\end{array}\right)
$$

where $A_{1}$ is Hurwitz stable, $x_{1} \in R^{n-1}$ and $x_{2} \in R$, and system (3.4) can be rewritten as

$$
\begin{gathered}
d x_{1}(t)=A_{1} x_{1}(t) d t+B_{1} u(t) d t+G_{1} x_{1}(t) d W_{t}, \\
d x_{2}(t)=b u(t) d t+g x_{2}(t) d W_{t}, \\
y(t)=C_{1} x_{1}(t)+x_{2}(t) .
\end{gathered}
$$

Note that $\left(x_{1}^{T}, x_{2}\right)=\left(0^{T}, 0\right)$ is an equilibrium point of PWM feedback system (3.6). 
Lemma 3.1 (The Itô isometry). Assume that $W(t)$ is a scalar wiener process, and If $\phi(t, \omega)$ is bounded and elementary, then

$$
E\left[\left(\int_{S}^{T} \phi(t, \omega) d W_{t}\right)^{2}\right]=E\left[\int_{S}^{T} \phi(t, \omega)^{2} d t\right] .
$$

Proof. Put $\Delta W_{j}=W_{t_{j+1}}-W_{t_{j}}$, then

$$
E\left[e_{i} e_{j} \Delta W_{i} \Delta W_{j}\right]= \begin{cases}0 & \text { if } i \neq j \\ E\left[e_{j}^{2}\right]\left(t_{j+1}-t_{j}\right) & \text { if } i=j\end{cases}
$$

Using that $e_{i} e_{j} \Delta W_{i}$ and $\Delta W_{j}$ are independent if $i<j$. Thus

$$
\begin{aligned}
E\left[\left(\int_{S}^{T} \phi(t, \omega) d W_{t}\right)^{2}\right] & =\sum_{i, j} E\left[e_{i} e_{j} \Delta W_{i} \Delta W_{j}\right]=\sum_{j} e_{j}^{2} \cdot\left(t_{i}-t_{j}\right) \\
& =E\left[\int_{S}^{T} \phi(t, \omega)^{2} d t\right]
\end{aligned}
$$

Theorem 3.2. Assume that the matrix $A_{1}$ in (3.6) is Hurwitz stable, $b>0$, and $C_{1} \neq 0$. Then the equilibrium $x_{e}=0$ of PWM feedback system (3.6) is the pth moment uniformly asymptotically stable in mean in the large whenever there exists a positive definite matrix $P=\left(\begin{array}{cc}P_{1} & 0 \\ 0 & \xi\end{array}\right)$ such that $M \beta<$ $\inf _{T_{k} \in[0, T]}\left(2 b \xi /\left(G_{2}\left(T_{k}\right)+b^{2} \xi+\max \left(0, \theta\left(T_{k}\right)\right)\right)\right.$ ), and there exists $a \delta>0$ (an upper bounded of $\delta$ will be given in the proof) such that $\|G\|<\delta$, where

$$
\begin{gathered}
\theta\left(T_{k}\right)=b^{2} \xi^{2} \lambda_{M}\left(C_{1}^{T} C_{1}\right)-b \xi \lambda_{m}\left(U\left(T_{k}\right) C_{1}+\left(U\left(T_{k}\right) C_{1}\right)^{T}\right)+\lambda_{M}\left(U\left(T_{k}\right) U^{T}\left(T_{k}\right)\right), \\
U\left(T_{k}\right)=\left(P_{1}-I\right)\left(\frac{I-e^{-A_{1} T_{k}}}{T_{k}} A_{1}^{-1} B\right) \\
G_{2}\left(T_{k}\right)=\left(\frac{I-e^{-A_{1} T_{k}}}{T_{k}} A_{1}^{-1} B\right)^{T}\left(P_{1}-I\right)\left(\frac{I-e^{-A_{1} T_{k}}}{T_{k}} A_{1}^{-1} B\right)
\end{gathered}
$$

Proof. Since $\left(E\|X\|^{p}\right)^{1 / p}$ is nondecreasing in $p$, the equilibrium $x_{e}=0$ is stable (resp., asymptotically stable, etc.) and implies that it is the $q$ th moment stable (resp., asymptotically stable, etc.) in mean for all $q<p$. Therefore, it suffices to prove the theorem for even integers.

Integrating (3.6), we have

$$
\begin{gathered}
x_{1}(t)=e^{A_{1}(t-k T)} x_{1}(k T)+\int_{k T}^{t} e^{A_{1}(t-s)} B_{1} u(s) d s+\int_{k T}^{t} e^{A_{1}(t-s)} G_{1} x_{1}(s) d W_{s} \\
x_{2}(t)=x_{2}(k T)+\int_{k T}^{t} b u(s) d s+\int_{k T}^{t} g x_{2}(s) d W_{s} .
\end{gathered}
$$


Therefore, when $t=k T+T$,

$$
\begin{aligned}
x_{1}(k T+T)= & e^{A_{1} T}\left(x_{1}(k T)-\int_{0}^{T_{k}} e^{-A_{1} s} B_{1} M \operatorname{sgn}\left(C_{1} x_{1}(k T)+x_{2}(k T)\right) d s\right) \\
& +\int_{k T}^{k T+T} e^{A_{1}(k T+T-s)} G_{1} x_{1}(s) d W_{s} \\
= & e^{A_{1} T}\left[\left(I-M \beta W\left(\tau_{k}\right) C_{1}\right) x_{1}(k T)-M \beta W_{1}\left(\tau_{k}\right) x_{2}(k T)\right] \\
& +\int_{k T}^{k T+T} e^{A_{1}(k T+T-s)} G_{1} x_{1}(s) d W_{s}, \\
x_{2}(k T+T)= & x_{2}(k T)-\int_{0}^{T_{k}} b M \operatorname{sgn}\left(C_{1} x_{1}(k T)+x_{2}(k T)\right) d s+\int_{k T}^{k T+T} g x_{2}(s) d W_{s} \\
= & -M \beta b \frac{T_{k}}{\tau_{k}} C_{1} x_{1}(k T)+\left(1-M \beta b \frac{T_{k}}{\tau_{k}}\right) x_{2}(k T)+\int_{k T}^{k T+T} g x_{2}(s) d W_{s}
\end{aligned}
$$

with

$$
\begin{gathered}
\tau_{k}=\beta\left|C_{1} x_{1}(k T)+x_{2}(k T)\right| \\
= \begin{cases}T_{k}, & T_{k}<T, \\
\geq T, & T_{k}=T,\end{cases} \\
W\left(\tau_{k}\right)= \begin{cases}0 & \tau_{k}=0, \\
\frac{I-e^{-A_{1} T_{k}}}{T_{k}} A_{1}^{-1} B & \tau_{k}<T, \\
\frac{I-e^{-A_{1} T}}{\tau_{k}} A_{1}^{-1} B=\frac{T}{\tau_{k}} W(T) & \tau_{k} \geq T .\end{cases}
\end{gathered}
$$

To simplify our notations, we let

$$
\begin{gathered}
H=\left[I-M \beta W\left(\tau_{k}\right)-M \beta W\left(\tau_{k}\right)\right], \\
Z(k T)=\int_{k T}^{k T+T} e^{A_{1}(k T+T-s)} G_{1} x_{1}(s) d W_{s},
\end{gathered}
$$


Abstract and Applied Analysis

$$
\begin{aligned}
& \tilde{Z}(k T)=\int_{k T}^{k T+T} g x_{2}(s) d W_{s}, \\
& \rho\left(\tau_{k}\right)= \begin{cases}0, & \tau_{k}=0, \\
\frac{T_{k}}{\tau_{k}}, & \tau_{k} \in(0, \infty),\end{cases} \\
& L\left(\tau_{k}\right)=\left[\begin{array}{ll}
-M \beta b \frac{T_{k}}{\tau_{k}} C_{1} & 1-M \beta b \frac{T_{k}}{\tau_{k}}
\end{array}\right] \\
& =\left[\begin{array}{lll}
-M \beta b \rho\left(\tau_{k}\right) C_{1} & 1-M \beta b \rho\left(\tau_{k}\right)
\end{array}\right] \text {. }
\end{aligned}
$$

Then (3.12) and (3.13) are reduced to

$$
\begin{aligned}
& x_{1}(k T+T)=e^{A_{1} T} H x(k T)+Z(k T), \\
& x_{2}(k T+T)=L\left(\tau_{k}\right) x(k T)+\tilde{Z}(k T) .
\end{aligned}
$$

Since $A_{1}$ is Hurwitz stable, then $e^{A_{1} T}$ is Schur stable. Therefore, there exists a positive definite matrix $P_{1}=P_{1}^{T}$ such that

$$
\left(e^{A_{1} T}\right)^{T} P_{1} e^{A_{1} T}-P_{1}=-I
$$

Choosing the quadratic Lyapunov function

$$
V: R^{n} \longrightarrow R^{+}, \quad V(x)=x^{T} P x,
$$

where

$$
P=\left(\begin{array}{cc}
P_{1} & 0 \\
0 & \xi
\end{array}\right), \quad P_{1}>0, \xi>0
$$

Then,

$$
\begin{aligned}
E[V(x(k T+T))]-E[V(x(k T))] \\
=E\left[x^{T}(k T+T) P x(k T+T)\right]-E\left[x^{T}(k T) P x(k T)\right] \\
=E\left[x_{1}^{T}(k T+T) P_{1} x_{1}(k T+T)\right]+E\left[x_{2}^{T}(k T+T) \xi x_{2}(k T+T)\right] \\
\quad-E\left[x^{T}(k T) P x(k T)\right] \\
=E\left[\left(e^{A_{1} T} H x(k T)+Z(k T)\right)^{T} P_{1}\left(e^{A_{1} T} H x(k T)+Z(k T)\right)\right]
\end{aligned}
$$




$$
\begin{aligned}
& +E\left[\left(L\left(\tau_{k}\right) x(k T)+\tilde{Z}(k T)\right)^{T} \xi\left(L\left(\tau_{k}\right) x(k T)+\tilde{Z}(k T)\right)\right] \\
& -E\left[x^{T}(k T) P x(k T)\right] \\
= & E\left[x^{T}(k T)\left(H^{T}\left(P_{1}-I\right) H+\xi L^{T}\left(\tau_{k}\right) L\left(\tau_{k}\right)-P\right) x(k T)\right] \\
& +2 E\left[Z^{T}(k T) P_{1} e^{A_{1} T} H x_{1}(k T)\right]+2 E\left[\tilde{Z}^{T}(k T) \xi x_{2}(k T)\right] \\
& +E\left[Z^{T}(k T) P_{1} Z(k T)\right]+E\left[\tilde{Z}^{T}(k T) \xi \tilde{Z}(k T)\right] \\
= & E\left[x^{T}(k T)\left(H^{T}\left(P_{1}-I\right) H+\xi L^{T}\left(\tau_{k}\right) L\left(\tau_{k}\right)-P\right) x(k T)\right] \\
& +2 E\left[h^{T}(k T) \Phi x(k T)\right]+E\left[h^{T}(k T) P h(k T)\right],
\end{aligned}
$$

where

$$
\begin{gathered}
x(k T)=\left(\begin{array}{c}
x_{1}(k T) \\
x_{2}(k T)
\end{array}\right), \quad h(k T)=\left(\begin{array}{c}
Z(k T) \\
\tilde{Z}(k T)
\end{array}\right), \\
\Phi=\left(\begin{array}{c}
P_{1} e^{A_{1} T} H \\
\xi L\left(\tau_{k}\right)
\end{array}\right)=\left(\begin{array}{cc}
P_{1} e^{A_{1} T}\left(I-M \beta W\left(\tau_{k}\right) C_{1}\right) & -M \beta P_{1} e^{A_{1} T} W\left(\tau_{k}\right) \\
-M \beta b \rho\left(\tau_{k}\right) \xi C_{1} & \xi-M \beta b \rho\left(\tau_{k}\right) \xi
\end{array}\right)=\Phi_{1}+\left(\begin{array}{ll}
0 & 0 \\
0 & \xi
\end{array}\right),
\end{gathered}
$$

with

$$
\Phi_{1}=\left(\begin{array}{cc}
P_{1} e^{A_{1} T}\left(I-M \beta W_{1}\left(\tau_{k}\right) C_{1}\right) & -M \beta P_{1} e^{A_{1} T} W_{1}\left(\tau_{k}\right) \\
M \beta b \rho\left(\tau_{k}\right) \xi & -M \beta b \rho\left(\tau_{k}\right) \xi
\end{array}\right) .
$$

Let

$$
\begin{aligned}
Q & =H^{T}\left(P_{1}-I\right) H+\xi L^{T}\left(\tau_{k}\right) L\left(\tau_{k}\right)-P \\
& =\left(\begin{array}{cc}
-I-M \beta\left(U\left(\tau_{k}\right) C_{1}+\left(U\left(\tau_{k}\right) C_{1}\right)^{T}\right)+\left(\gamma+2 M \beta b \rho\left(\tau_{k}\right) \xi\right) C_{1} C_{1}^{T} & D_{12} \\
D_{12}^{T} & \gamma
\end{array}\right),
\end{aligned}
$$

where

$$
\begin{gathered}
r=M^{2} \beta^{2}\left[G_{2}\left(\tau_{k}\right)+b^{2} \rho^{2}\left(\tau_{k}\right) \xi\right]-2 M \beta b \rho\left(\tau_{k}\right) \xi, \\
D_{12}=-M \beta U\left(\tau_{k}\right)+\left[\gamma+M \beta b \rho\left(\tau_{k}\right) \xi\right] C_{1}^{T},
\end{gathered}
$$


with

$$
\begin{gathered}
U\left(\tau_{k}\right)=\left(P_{1}-I\right) W\left(\tau_{k}\right), \\
G_{2}\left(\tau_{k}\right)=W^{T}\left(\tau_{k}\right)\left(P_{1}-I\right) W\left(\tau_{k}\right) .
\end{gathered}
$$

Then (3.21) is reduced to

$$
\begin{aligned}
& E[V(x(k T+T))]-E[V(x(k T))] \\
&= E\left[x^{T}(k T) Q x(k T)\right]+2 E\left[h^{T}(k T) \Phi_{1} x(k T)\right] \\
&+E\left[h^{T}(k T) P h(k T)\right]+2 \xi E\left[\tilde{Z}(k T) x_{2}(k T)\right] .
\end{aligned}
$$

By elementary transformation of matrix, we have

$$
Q=\left(\begin{array}{cc}
I & \frac{D_{12}}{r} \\
0 & 1
\end{array}\right) D\left(\begin{array}{cc}
I & 0 \\
\frac{D_{12}^{T}}{r} & 1
\end{array}\right)
$$

where

$$
D=-\left(\begin{array}{cc}
D_{11} & 0 \\
0 & -\gamma
\end{array}\right)
$$

with

$$
\begin{aligned}
D_{11}=\frac{1}{\gamma}[ & \gamma I+M^{2} \beta^{2}\left(U\left(\tau_{k}\right) U^{T}\left(\tau_{k}\right)-M^{2} \beta^{2} b \rho\left(\tau_{k}\right) \xi\left(U\left(\tau_{k}\right) C_{1}+\left(U\left(\tau_{k}\right) C_{1}\right)^{T}\right)\right) \\
& \left.+M^{2} \beta^{2} b^{2} \rho^{2}\left(\tau_{k}\right) \xi^{2} C_{1}^{T} C_{1}\right] .
\end{aligned}
$$

Let $\lambda_{m}(\cdot)$ and $\lambda_{M}(\cdot)$ denote the minimum and maximum eigenvalues of a matrix, respectively. It is obvious that $D$ is a negative definite matrix if $D_{11}$ is positive definite matrix with $\gamma<0$. Note that, with $r<0$, we have

$$
\begin{aligned}
\lambda_{m}\left(D_{11}\right) \geq 1+(M \beta)^{2} \gamma^{-1}\{ & \lambda_{M}\left[U\left(\tau_{k}\right) U^{T}\left(\tau_{k}\right)\right] \\
& \left.-b \rho\left(\tau_{k}\right) \xi \lambda_{m}\left[U\left(\tau_{k}\right) C_{1}+\left(U\left(\tau_{k}\right) C_{1}\right)^{T}\right]+b^{2} \rho^{2}\left(\tau_{k}\right) \xi^{2} \lambda_{M}\left[C_{1}^{T} C_{1}\right]\right\} .
\end{aligned}
$$


Therefore, the claim that $D_{11}$ is positive definite is true if

$$
\begin{aligned}
(M \beta)\left(G_{2}\left(\tau_{k}\right)\right. & \left.+b^{2} \rho^{2}\left(\tau_{k}\right) \xi\right)-2 b \rho\left(\tau_{k}\right) \xi \\
+(M \beta)\{ & \lambda_{M}\left[U\left(\tau_{k}\right) U^{T}\left(\tau_{k}\right)\right]-b \rho\left(\tau_{k}\right) \xi \lambda_{m}\left[U\left(\tau_{k}\right) C_{1}+\left(U\left(\tau_{k}\right) C_{1}\right)^{T}\right] \\
& \left.+b^{2} \rho^{2}\left(\tau_{k}\right) \xi^{2} \lambda_{M}\left[C_{1}^{T} C_{1}\right]\right\} \leq 0
\end{aligned}
$$

with

$$
M \beta\left(G_{2}\left(\tau_{k}\right)+b^{2} \rho^{2}\left(\tau_{k}\right) \xi\right)-2 b \rho\left(\tau_{k}\right) \xi<0
$$

That is,

$$
\begin{aligned}
M \beta & <\inf _{\tau_{k} \in[0, \infty]} \frac{2 b \rho\left(\tau_{k}\right) \xi}{G_{2}\left(\tau_{k}\right)+b^{2} \xi+\max \left(0, \theta\left(\tau_{k}\right)\right)} \\
& =\inf _{T_{k} \in[0, T]} \frac{2 b \xi}{G_{2}\left(T_{k}\right)+b^{2} \xi+\max \left(0, \theta\left(T_{k}\right)\right)}
\end{aligned}
$$

where

$$
\begin{aligned}
\theta\left(\tau_{k}\right)= & b^{2} \rho^{2}\left(\tau_{k}\right) \xi^{2} \lambda_{M}\left(C_{1}^{T} C_{1}\right)-b \rho\left(\tau_{k}\right) \xi \lambda_{m}\left(U\left(\tau_{k}\right) C_{1}+\left(U\left(\tau_{k}\right) C_{1}\right)^{T}\right) \\
& +\lambda_{M}\left(U\left(\tau_{k}\right) U^{T}\left(\tau_{k}\right)\right), \\
\theta\left(T_{k}\right)= & b^{2} \xi^{2} \lambda_{M}\left(C_{1}^{T} C_{1}\right)-b \xi \lambda_{m}\left(U\left(T_{k}\right) C_{1}+\left(U\left(T_{k}\right) C_{1}\right)^{T}\right) \\
& +\lambda_{M}\left(U\left(T_{k}\right) U^{T}\left(T_{k}\right)\right) .
\end{aligned}
$$

Thus, whenever $M \beta$ satisfies (3.34) for some positive constant $\xi$, then $D$ is negative definite matrix, and, therefore, $Q$ is negative definite matrix. By elementary transform of matrix, one observes that

$$
\Phi_{1}=\left(\begin{array}{cc}
I & P_{1} e^{A_{1} T} \frac{W\left(\tau_{k}\right)}{b \rho\left(\tau_{k}\right) \xi} \\
0 & 1
\end{array}\right) \Phi_{2}\left(\begin{array}{cc}
I & 0 \\
C_{1} & 1
\end{array}\right)
$$

where

$$
\Phi_{2}=\left(\begin{array}{cc}
P_{1} e^{A_{1} T} & 0 \\
0 & -M \beta \rho\left(\tau_{k}\right) b \xi
\end{array}\right) .
$$

So, we have $\left\|\Phi_{1}\right\| \leq\left\|\Phi_{2}\right\|$. 
Now we establish an estimate for $h(k T)$. Let $\mu>0$ be arbitrary. We will show that there exists $\delta>0$ such that $E\|h(k T)\|^{2}<\mu E\|x(k T)\|^{2}$ whenever $\|G\|<\delta$.

For $t \in[k T, k T+T]$, we have

$$
x(t)=x(k T)+\int_{k T}^{t}(A x(s)+B u(s)) d s+\int_{k T}^{t} G x(s) d W_{s} .
$$

Note that

$$
E\left(\int_{k T}^{t}\|B\||u(s)| d s\right)^{2} \leq M^{2} \beta^{2}\|B\|^{2}\|C\|^{2} E\left[\|x(k T)\|^{2}\right] .
$$

Then,

$$
\begin{aligned}
\frac{E\left[\|x(t)\|^{2}\right]}{4} \leq & E\left[\|x(k T)\|^{2}\right]+E\left[\left\|\int_{k T}^{t} A x(s) d s\right\|^{2}\right] \\
& +E\left[\left(\int_{k T}^{t}\|B\||u(s)| d s\right)^{2}\right]+E\left[\left(\int_{k T}^{t} G x(s) d W_{s}\right)^{T}\left(\int_{k T}^{t} G x(s) d W_{s}\right)\right] .
\end{aligned}
$$

By Lemma 3.1, one observes that

$$
E\left[\left(\int_{k T}^{t} G x(s) d W_{s}\right)^{T}\left(\int_{k T}^{t} G x(s) d W_{s}\right)\right]=E\left[\int_{k T}^{t} x^{T}(s) G^{T} G x(s) d s\right] .
$$

Then (3.40) is reduced to

$$
\frac{E\left[\|x(t)\|^{2}\right]}{4} \leq K_{0} E\left[\|x(k T)\|^{2}\right]+\left(\|A\|^{2}+\|G\|^{2}\right) \int_{k T}^{t} E\left[\|x(s)\|^{2}\right] d s,
$$

where $K_{0}=1+M^{2} \beta^{2}\|B\|^{2}\|C\|^{2}$.

By Gronwall inequality, we have

$$
\begin{aligned}
E\left[\|x(t)\|^{2}\right] & \leq 4 K_{0} e^{\left(\|A\|^{2}+\|G\|^{2}\right)(t-k T)} E\left[\|x(k T)\|^{2}\right] \\
& \leq 4 K_{0} e^{\left(\|A\|^{2}+\|G\|^{2}\right) T} E\left[\|x(k T)\|^{2}\right] .
\end{aligned}
$$


Thus,

$$
\begin{aligned}
E\left[\|h(k T)\|^{2}\right]= & E\left[\left(\int_{k T}^{k T+T} e^{A_{1}(k T+T-s)} G_{1} x_{1}(s) d W_{s}\right)^{T}\left(\int_{k T}^{k T+T} e^{A_{1}(k T+T-s)} G_{1} x_{1}(s) d W_{s}\right)\right] \\
& +E\left[\left(\int_{k T}^{k T+T} g x_{2}(s) d W_{s}\right)^{T}\left(\int_{k T}^{k T+T} g x_{2}(s) d W_{s}\right)\right] \\
= & E\left[\int_{k T}^{k T+T} x_{1}^{T}(s) G_{1}^{T} e^{A_{1}^{T}(k T+T-s)} e^{A_{1}(k T+T-s)} G_{1} x_{1}(s) d s\right]+E\left[\int_{k T}^{k T+T} g^{2} x_{2}^{2}(s) d s\right] \\
= & E\left[\int_{k T}^{k T+T} x^{T}(s) \tilde{G}^{T} \tilde{G} x(s) d s\right] \\
\leq & K_{1}\|G\|^{2} \int_{k T}^{k T+T} E\left[\|x(s)\|^{2}\right] d s \\
\leq & 4 K_{0} K_{1}\|G\|^{2} e^{\left(\|A\|^{2}+\|G\|^{2}\right) T} E\left[\|x(k T)\|^{2}\right],
\end{aligned}
$$

where

$$
\begin{gathered}
\tilde{G}=\left(\begin{array}{cc}
e^{A_{1}(k T+T-s)} & 0 \\
0 & g
\end{array}\right), \\
K_{1}=\max _{s \in[0, T]}\left\|e^{A_{1}^{T} s} e^{A_{1} s}\right\|, \quad K=4 K_{0} K_{1} e^{\|A\|^{2} T} .
\end{gathered}
$$

Thus, there exists a $\delta>0$ such that $K\|G\|^{2} e^{\|G\|^{2} T}<\mu$ whenever $\|G\|<\delta$. Choosing $\mu>0$, so that $c(\mu)=1-2\left(\left\|\Phi_{2}\right\|+\xi\right) \sqrt{\mu}-\|P\| \mu>0$. Then, we obtain that

$$
E[V(x(k T+T))]-E[V(x(k T))] \leq-\frac{c(\mu)}{\lambda_{M}(P)} E[V(x(k T))]
$$

Therefore, $E[V(x(k T+T))]-E[V(x(k T))]$ is negative definite.

If we define $\widetilde{M}=\{0\}$ and $\widetilde{S}=\left\{\widetilde{x}: \widetilde{x}\left(t, \tilde{a}, t_{0}\right)=E\left[V\left(x\left(t, a, t_{0}\right)\right)\right]\right\}$, then we have determined in an unambiguous way a deterministic dynamical system $\left\{R^{+}, R^{+}, R^{+}, S\right\}$, corresponding to the stochastic dynamical system $\left\{R^{+}, R^{n}, R^{n}, S_{(4)}\right\}\left(S_{(4)}\right.$ is the trivial solution set of system (3.6)). In this case $M$ is an invariant set for $\left\{R^{+}, R^{+}, R^{+}, S\right\}$.

In fact, $\{E V(x(k T)\}$ is uniformly asymptotically stable (see, e.g., [21]). For $t \in[k T, k T+$ $T]$, we have

$$
E[V(x(t))] \leq \lambda_{M}(P) K e^{\|G\|^{2} T} E\|x(k T)\|^{2} \leq \frac{\lambda_{M}(P)}{\lambda_{m}(P)} V(x(k T)) .
$$


This implies that $E[V(x(t))]$ in the interval $[k T, k T+T]$ is bounded by $r E V(x(k T))$, where $r>0$ is constant. Therefore, $E[V(x(t))]$ converges to the origin simultaneously with $E V(x(k T))$. We conclude that the trivial solution of deterministic system $\widetilde{S}$ is asymptotically stable. Now recall that $(\widetilde{S}, \widetilde{M})$ is uniformly stable if, for every $\varepsilon>0, t_{0} \in R^{+}$, there exists a $\delta=\delta(\varepsilon)>0$ such that $\tilde{x}\left(t, \tilde{a}, t_{0}\right)<\varepsilon$ for all $t \in R_{\tilde{a}, t_{0}}^{+}=R_{a, t_{0}}^{+}$and for all $\tilde{x}\left(\cdot, \tilde{a}, t_{0}\right) \in \widetilde{S}$, whenever $\tilde{a}<\delta$.

Since $\tilde{x}\left(t, \tilde{a}, t_{0}\right)=E V(x(t))$, thus, $(\widetilde{S}, \widetilde{M})$ is uniformly stable means that, for every $\varepsilon>$ $0, t_{0} \in R^{+}$, there exists a $\delta=\delta(\varepsilon)>0$ such that $E V(x(t))<\varepsilon$ for $t \in R_{a, t_{0}}^{+}$and for all $x\left(\cdot, a, t_{0}\right) \in$ $S$, whenever $E V(a)<\delta$, since $E V(x(t)) / \lambda_{M}(P) \leq E\|x(t)\|^{2} \leq E V(x(t)) / \lambda_{m}(P)$; hence, for every $\varepsilon>0, t_{0} \in R^{+}$, for any process $x\left(\cdot, \cdot, a, t_{0}\right) \in S$, we have, for $t \in R_{a, t_{0}}^{+}, E\|x(t)\|^{2} \leq$ $\varepsilon / \lambda_{m}(P)$, whenever $\|a\|<\delta_{1}$, where $\delta_{1}=\delta / \lambda_{M}(P)$. This is precisely the definition of uniform stability in the mean square of the trivial solution $x_{e}=0$ of $S_{(4)}$. Therefore, the uniform stability of $(\widetilde{S}, \widetilde{M})$ is equivalent to the uniform stability in the mean square of $\left(S_{(4)},\left\{x_{e}\right\}\right)$.

We can now conclude that the equilibrium $x_{e}=0$ of PWM feedback system (3.6) is uniformly asymptotically stable in mean square.

For $p=2 q, q \geq 1$, we have

$$
\begin{aligned}
& E\left[V(x(k T+T))^{q}\right]-E\left[V(x(k T))^{q}\right] \\
&=E\left[(V(x(k T+T))-V(x(k T))) \times\left(V(x(k T+T))^{q-1}+\cdots+V(x(k T))^{q-1}\right)\right] \\
& \leq E\left[\left(-\frac{1}{\lambda_{M}(P)} V(x(k T))+2 E h^{T}(k T) \Phi x(k T)+E h^{T}(k T) P h(k T)\right)\right. \\
&\left.\times\left(V(x(k T+T))^{q-1}+\cdots+V(x(k T))^{q-1}\right)\right] \\
& \leq-\frac{1}{\lambda_{M}(P)} E\left[V(x(k T))^{q}\right]+E\left[\left(2 h^{T}(k T) \Phi x(k T)+2 h^{T}(k T) \Phi h(k T)\right)\right. \\
&\left.\times\left(V(x(k T+T))^{q-1}+\cdots+V(x(k T))^{q-1}\right)\right] .
\end{aligned}
$$

It can easily be verified that the second expectation in the above inequality can always be chosen to be less than $\mu E V(x(k T))^{q}$ for arbitrary $\mu$, when $\|G\|$ is sufficiently small. Similarly as in [22], we can show that $E V(x(t))^{q}$ is bounded by $E V(x(k T))^{q}$ when $t \in[k T, k T+T]$, where $a>0$ is a constant.

The rest of the proof of the $p$ th moment uniform asymptotic stability of the trivial solution of (3.6) with $p=2 q$ proceeds similarly as the proof of uniform asymptotic stability in the mean square of the trivial solution of (3.6).

Therefore, we have shown that the trivial solution of system (3.6) is the $p$ th moment uniformly asymptotically stable in mean for even integers, and, hence, we conclude the proof for all $p>0$.

Remark 3.3. The upper bound of $M \beta$ is given by (3.34) that can easily be computed and optimized. A simple procedure is presented in [20] to accomplish that. We will employ this procedure in a specific example in Section 4.

Theorem 3.4. Assume that $A_{1}$ is Hurwitz stable, $C_{1}=0$, and $b>0$. Then the trivial solution of the PWM feedback system (3.6) is the pth moment uniformly asymptotically stable in mean whenever 
$M \beta<2 / b$, and there exists $a \delta>0$ (an upper bounded of $\delta$ will be given in the proof of Theorem 3.2) such that $\|G\|<\delta$.

Proof. When $C_{1}=0$, we have

$$
D_{11}=\frac{1}{\gamma}\left[\gamma I+M^{2} \beta^{2} U\left(\tau_{k}\right) U^{T}\left(\tau_{k}\right)\right]
$$

If $\lambda_{m}\left(D_{11}\right)>0, D_{11}$ is positive definite matrix. And if $-\gamma>0$, then $D$ is positive negative definite matrix. Therefore, whenever $\lambda_{m}\left(D_{11}\right)>0$ and $\gamma<0$, it can easily be verified that

$$
\left(M \beta b^{2}-2 b\right) \xi+\lambda_{M}\left(U\left(\tau_{k}\right) U^{T}\left(\tau_{k}\right)\right)+G_{2}\left(\tau_{k}\right)>0 .
$$

If $M \beta<2 / b$, there exists $\xi$ such that (3.50) and $\lambda<0$ are true for all $M \beta$. Hence, whenever $M \beta<2 / b$ for some positive constant $\xi$, then $Q$ is negative definite matrix.

The rest of the proof of the uniformly $p$ th moment asymptotic stability in mean of the trivial solution of (3.6) proceeds similarly as that of Theorem 3.2.

Remark 3.5. From stability results of Theorem 3.4, we can obtain when upper bound of the parameters of PWM feedback system $(M \beta)$ is sufficiently large, and if we choose that $b$ is sufficiently small, then the trivial solution of the system (3.6) is the $p$ th moment uniformly asymptotic stability in mean.

Next, we consider PWM feedback system with additive noise in the plant, described by equations of the form. We have

$$
\begin{gathered}
d x_{1}(t)=A_{1} x_{1}(t) d t+B_{1} u(t) d t+\varepsilon_{1} x_{1}(t) d W_{t}, \\
d x_{2}(t)=b u(t) d t+\varepsilon_{2} x_{2}(t) d W_{t}, \\
y(t)=C_{1} x_{1}(t)+x_{2}(t),
\end{gathered}
$$

where $\varepsilon_{1} \in R^{n-1}, \varepsilon_{2} \in R$ have positive components. We let $\varepsilon=\left(\begin{array}{ll}\varepsilon_{1} & \varepsilon_{2}\end{array}\right) \in R^{n}$ denote the magnitude of the random noise. A block diagram of system (3.51) is shown in Figure 2.

Theorem 3.6. Assume that $A_{1}$ is Hurwitz stable, and the solution processes of the PWM feedback system (3.6) are the pth moment uniformly ultimately bounded provided by $b>0$, all $M \beta \in(0, \infty)$ (for random disturbance). When $b \leq 0$, the solution processes are unbounded.

Proof. The proof of Theorem 3.6 is similar as that of [20].

To end this section, we present an algorithm for computing stability bound.

\section{Stability Bound Algorithm}

An upper bound of $M \beta$ that satisfies (3.34) can be computed and optimized in the following manner. 


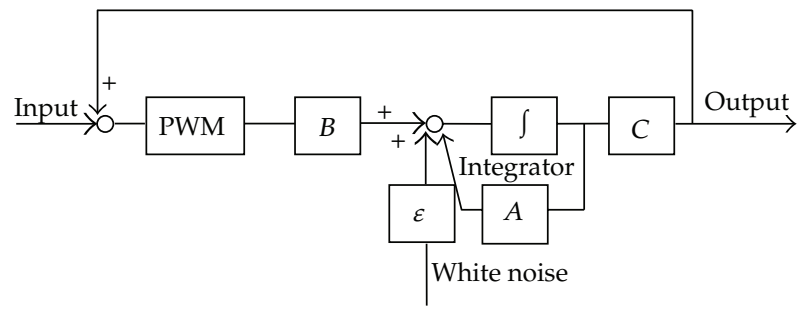

Figure 2: Block diagram of PWM feedback systems subjected to additive disturbances.

(S1) Determine the matrix $P_{1}$ by solving $\left(e^{A_{1} T}\right)^{T} P_{1} e^{A_{1} T}-P_{1}=-I$.

(S2) Choose a precision level $\delta>0$ and a correspondingly dense partition of the interval $[0, T]$, and say the set $\left\{t_{0}=0, t_{1}, \ldots, t_{N}=T\right\}$, where $0<t_{j+1}-t_{j}<\delta, j=$ $0,1,2, \ldots, N-1$.

(S3) For each $i, j=0,1,2, \ldots, N-1$, calculate

$$
\begin{gathered}
W\left(t_{j}\right)=\frac{I-e^{-A_{1} t_{j}}}{t_{j}} A_{1}^{-1} B, \quad G_{2}\left(t_{j}\right)=W\left(t_{j}\right)^{T}\left(P_{1}-I\right) W\left(t_{j}\right), \\
U\left(t_{j}\right)=\left(P_{1}-I\right) W\left(t_{j}\right) .
\end{gathered}
$$

(S4) Search for the largest $M \beta$ such that

$$
M \beta<\inf _{t_{j} \in[0, \infty]} \frac{2 b \rho\left(t_{j}\right) \xi}{G_{2}\left(t_{j}\right)+b^{2} \xi+\max \left(0, \theta\left(t_{j}\right)\right)}
$$

where

$$
\theta\left(t_{j}\right)=b^{2} \xi^{2} \lambda_{M}\left(C_{1}^{T} C_{1}\right)-b \xi \lambda_{m}\left(U\left(t_{j}\right) C_{1}+\left(U\left(t_{j}\right) C_{1}\right)^{T}\right)+\lambda_{M}\left(U\left(t_{j}\right) U^{T}\left(t_{j}\right)\right) .
$$

(S5) Repeat steps $1-4$, using finer partition of the interval $[0, T]$ until there is no further significant improvement for $M \beta_{\text {opt }}$.

\section{Examples}

Example 4.1. Consider the system (3.6) with second-order critical stable plant described by transfer function $T(s)=k / s(1+(s / a)), a>0$. The state space representation of this system is given by

$$
A=\left(\begin{array}{cc}
-a & 0 \\
0 & 0
\end{array}\right), \quad B=\left(\begin{array}{l}
k \\
k
\end{array}\right), \quad C=\left[\begin{array}{ll}
1 & 1
\end{array}\right]
$$


Hence $A_{1}=-a, B_{1}=k, C_{1}=1$, and $P_{1}, W\left(T_{k}\right), U\left(T_{k}\right)$ in Theorem 3.2 are calculated in this case as

$$
\begin{gathered}
P_{1}=\frac{1}{1-e^{-2 a T}}, \quad W\left(T_{k}\right)=\frac{\left(e^{a T_{k}}-1\right) k}{a T_{k}}, \\
U\left(T_{k}\right)=\frac{k\left(e^{a T_{k}}-1\right)}{a T_{k}\left(e^{2 a T}-1\right)}, \quad G_{2}\left(T_{k}\right)=\frac{k^{2}\left(e^{a T_{k}}-1\right)^{2}}{a^{2} T_{k}^{2}\left(e^{2 a T}-1\right)}, \\
\theta\left(T_{k}\right)=k^{2} \xi^{2}-\frac{2 k^{2}\left(e^{a T_{k}}-1\right)}{a T_{k}\left(e^{2 a T}-1\right)} \xi+\frac{k^{2}\left(e^{a T_{k}}-1\right)^{2}}{a^{2} T_{k}^{2}\left(e^{2 a T}-1\right)^{2}} \\
=k^{2}\left(\xi-\frac{e^{a T_{k}}-1}{a T_{k}\left(e^{2 a T}-1\right)}\right)^{2} \geq 0 .
\end{gathered}
$$

Choosing $\xi=1 / a T\left(1+e^{-a T}\right)$, it can be seen that the stability bound is given by

$$
M \beta<\frac{1}{\left(\left(\left(e^{a T}-1\right) / a T\left(e^{a T}+1\right)\right)+(1 / 2)\right) k} .
$$

Assume that $M=1$, and then the condition for uniformly asymptotic stability in $p$ th mean is given by

$$
\frac{1}{k \beta}>\frac{1}{a T}-\frac{2}{a T\left(e^{a T}+1\right)}+\frac{1}{2}
$$

In Example 4.1, we obtain the stability results depicted in Figure $3\left(k \beta_{\text {opt }}\right.$ versus $\left.a T\right)$.

Example 4.2. Consider PWM feedback system (3.6) with transfer function $T(s)=1 / s(1+s)$. The state space representation of this system is given by

$$
A=\left(\begin{array}{cc}
-1 & 0 \\
0 & 0
\end{array}\right), \quad B=\left(\begin{array}{l}
1 \\
1
\end{array}\right), \quad C=\left[\begin{array}{ll}
1 & 1
\end{array}\right]
$$

Letting $\delta=0.01$, and assuming $T=1$, we obtain the estimation of the upper bound of $M \beta$, that is, $M \beta \leq 1.0394$. For $M \beta \in(0,1.0394)$, we compute $\mu_{\max }$ such that $c(\mu)=1-2\left(\left\|R_{2}\right\|+\right.$ $\xi) \sqrt{\mu}-\|P\| \mu>0$ is true for all $\mu<\mu_{\max }$. Next, we compute $\delta_{\max }$ such that $K \delta^{2} e^{\delta^{2} T}<\mu_{\max }$ is true for all $\delta<\delta_{\max }$, where $K=4 K_{1}\left(1+M^{2} \beta^{2}\|B\|^{2}\|C\|^{2}\right) e^{\|A\|^{2} T}$ and $K_{1}=\max _{s \in[0, T]}\left\|e^{A_{1}^{T} s} e^{A_{1} s}\right\|$. In Figure 4, we depict the estimates of the upper bound $\delta_{\max }$ of $\|G\|$ versus $M \beta$.

We observe that $\delta_{\max }$ decreases as $M \beta$ increases. When the states are sufficiently far away from the origin so that $T_{k}=T$, and the output of pulse-width modulator is either $+M$ or $-M$. Therefore, as $M$ increases (for fixed $\beta$ ), the maximum $\|G\|$ allowable to ensure the $p$ th moment uniform asymptotic stability in mean will decrease; besides, if disturbance of the feedback system (3.6) is increase (less than $\delta_{\max }$ ), the trivial solution of system (3.6) is the $p$ th moment uniformly asymptotically stable in mean by decreasing the value of $M \beta$, as shown in Figure 4. 


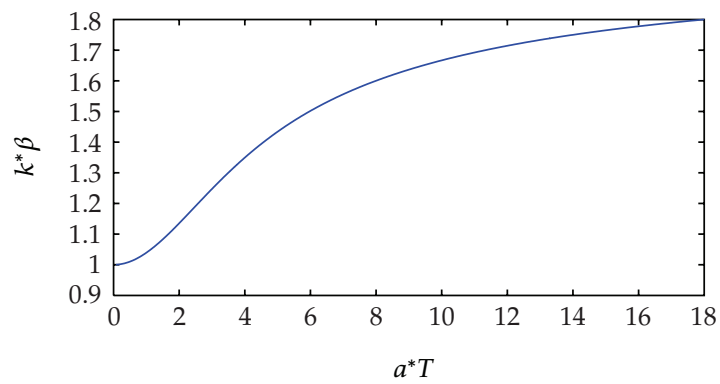

Figure 3: Estimates of upper bounds for $k \beta$ for the second-order PWM feedback system of Example 4.1.

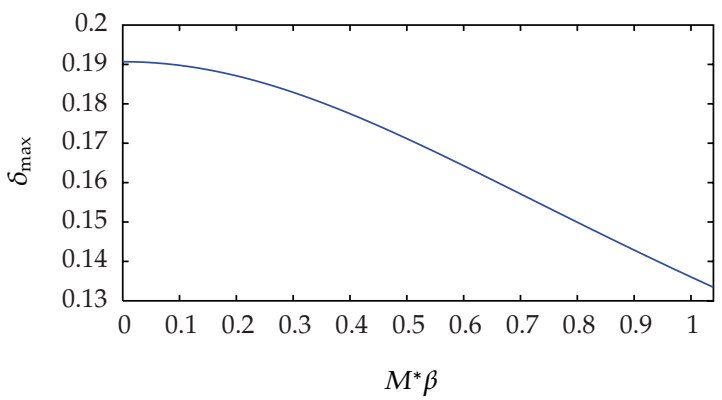

Figure 4: Upper bounds for $\|G\|$ when $M \beta \in(0,1.0394)$.

In Figure 5, we plot the sample response of $x=\left(\begin{array}{ll}x_{1} & x_{2}\end{array}\right)^{T}$ with $M=0.2, T=\beta=1, G=$ $\left(\begin{array}{cc}\left.\begin{array}{cc}0.02 & 0 \\ 0 & 0.006\end{array}\right) \text { and } x(0) \\ a\end{array}\right)\left(\begin{array}{ll}2 & 0.5\end{array}\right)^{T}$. We generated 100 sample responses of $x(t)$ and computed the average $\overline{\|x(t)\|}$ of $\|x(t)\|$. Figure 6 shows the average $\overline{\|x(t)\|}$ tending to zero as time $t$ increases. However, $\overline{\|x(t)\|}$ does not diminish entirely to zero since it is an approximation to the mean $E\|x(t)\|$.

\section{Conclusions}

We studied the critical case of PWM feedback systems with random perturbations and establish several Lyapunov and Lagrange criteria for the $p$ th moment uniform asymptotical stability in mean, and then we presented an algorithm to compute the upper bound for the parameters of PWM and finally gave two numerical examples to verify the effectiveness of theoretical results. We characterized the relationship between the parameters of PWM and the coefficients of state vectors of the feedback systems and showed that when the random disturbance is sufficiently small such that PWM feedback system is the $p$ th moment globally asymptotically stable in mean provided that the upper bounds of parameters of pulse-width modulator are selected properly. 


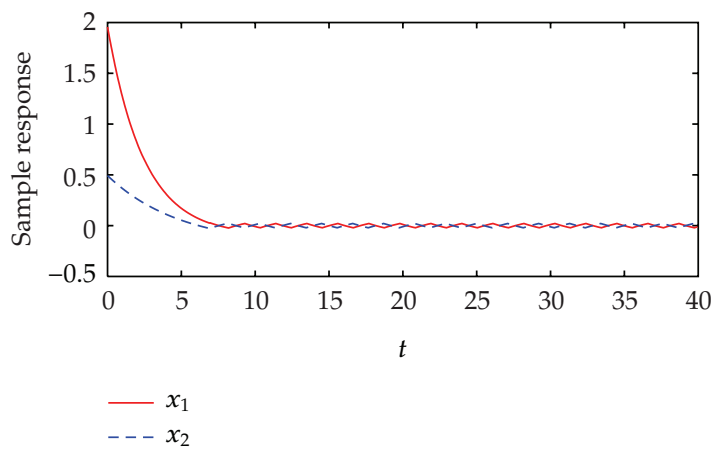

Figure 5: Sample response of PWM feedback system (3.6) in Example 4.2.

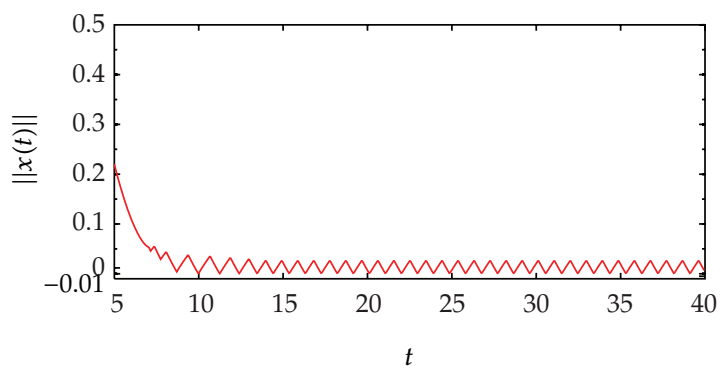

Figure 6: $\|x(t)\|$ of PWM feedback system (3.6) in Example 4.2.

\section{Acknowledgment}

This work was supported by the National Natural Science Foundation of China under Grant no. 60974020 and the Fundamental Research Funds for the Central Universities of China under Grant nos. CDJZR10100015 and CDJZR10185501.

\section{References}

[1] I. Takahashi, "A new control of PWM inverter wave form for minimum loss operation of an induction motor drive," IEEE Transactions on Industrial Electronics, vol. 21, no. 4, pp. 580-587, 1985.

[2] G. Heinle, "The structure of optimized pulse patterns," in Proceedings of the 5th European Conference on Power Electronics and Applications (EPE'93), pp. 378-383, Brighton, UK, 1993.

[3] J. Holt, "Pulse width modulation-a survey," IEEE Transactions on Industrial Electronics, vol. 39, no. 5, pp. 410-420, 1992.

[4] J. -C. Bor and C. -Y. Wu, "Realization of the CMOs pulse width-modulation(PWM) neural network with on-chip learning," IEEE Transactions on Circuits and Systems, vol. 45, no. 1, 1998.

[5] Z. Hui and B. Michael, "A new partly unsymmetrical PWM technique for harmopnic compensators," in Proceedings of the 11th European Conference on Power Electronics and Applications (EPE '05), Dresdnen, Germany, 2005.

[6] G. J. Murphy and S. H. Wu, "A stability criterion for pulse-width-modulated feedback control systems," Transactions on Automatic Control, vol. 9, pp. 434-441, 1964.

[7] A. Balestrino, A. Eisinberg, and L. Sciavicco, "A generalised approach to the stability analysis of PWM feedback control systems," Journal of the Franklin Institute, vol. 298, pp. 45-58, 1974.

[8] L. Hou and A. N. Michel, "Stability analysis of pulse-width-modulated feedback systems," Automatica, vol. 37, no. 9, pp. 1335-1349, 2001. 
[9] R. A. Skoog, "on the stability of pulse width modulated feedback systems," IEEE Transactions on Automatic Control, vol. AC-13, no. 5, 1968.

[10] A. Halanar̆, "Positive definite kernels and the stability of automatic systems," Revue Roumaine de Mathématiques Pures et Appliquées, vol. 9, pp. 751-765, 1964 (Russian).

[11] V. M. Kuntsevich and N. Yu. Chekhovei, Nonlinear Systems with Pulse Frequency and Pulse Width Modulation, Tekhnika, Kiev, Ukraine, 1970.

[12] L. Hou, Qualitative analysis of discontinuous deterministic and stochastic dynamical systems [Ph.D. dissertation], University of Notre Dame, 2000.

[13] V.-M. Popov, "Absolute stability of nonlinear systems of automatic control," Automation and Remote Control, vol. 22, no. 8, pp. 961-979, 1961.

[14] S. C. Gupta and E. I. Jury, "Statistical study of pulse-width modulated control systems," The Journal of The Franklin Institute, vol. 273, no. 4, pp. 292-321, 1962.

[15] H. Y. Sun, W. H. Zhang, and M. Li, "Stability and stabilization of stochastic systems by feedback control," in Proceedings of the 8th IEEE World Congress on Intelligent Control and Automation, July 2010.

[16] L. Liu, Y. Shen, and F. Jiang, "The almost sure asymptotic stability and $p$ th moment asymptotic stability of nonlinear stochastic differential systems with polynomial growth," Transactions on Automatic Control, vol. 56, no. 8, pp. 1985-1990, 2011.

[17] Y. Wang and S. Boyd, "Performance bounds and suboptimal policies for linear stochastic control via LMIs," International Journal of Robust and Nonlinear Control, vol. 21, no. 14, pp. 1710-1728, 2011.

[18] P. Balasubramanian and M. Ali, "stochastic stability of uncertain fuzzy recurrent neural networks with Markov jumping parameters," International Journal of Computer Mathematic, vol. 88, no. 5, pp. 892-904, 2011.

[19] L. Hou, "Stability analysis of pulse-width-modulated feedback systems with type 2 modulation: the critical case," in Proceedings of the 39th IEEE Conference on Decision and Control, Sydney, Australia, 2005.

[20] L. Hou and A. N. Michel, "Moment stability of pulse-width-modulated feedback systems subjected to random disturbances," in Proceedings of the 39th IEEE Conference on Decision and Control, Sydney, Australia, December 2000.

[21] L. Hou and A. N. Michel, "Moment stability of discontinuous dynamical systems," in Proceedings of the American Control Conference, pp. 3807-3811, Chicago, Ill, USA, June 2000.

[22] A. N. Michel and K. Wang, Qualitative Theory of Dynamical Systems, vol. 186 of Monographs and Textbooks in Pure and Applied Mathematics, Marcel Dekker, New York, NY, USA, 1995. 


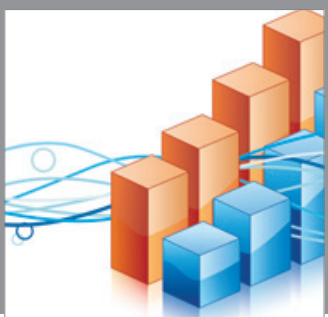

Advances in

Operations Research

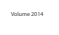

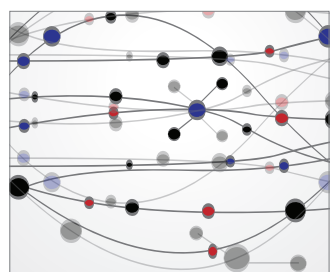

\section{The Scientific} World Journal
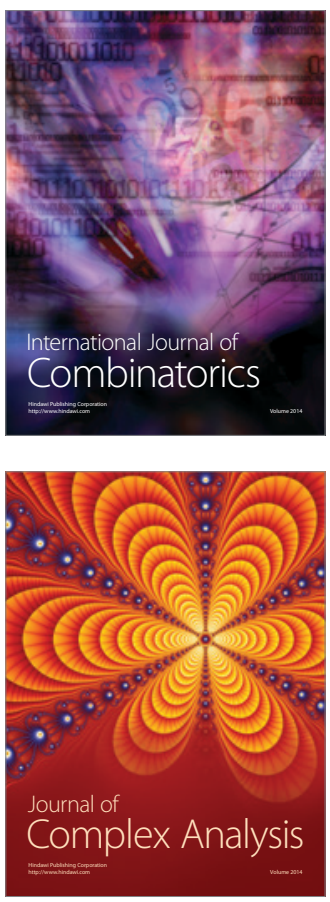

International Journal of

Mathematics and

Mathematical

Sciences
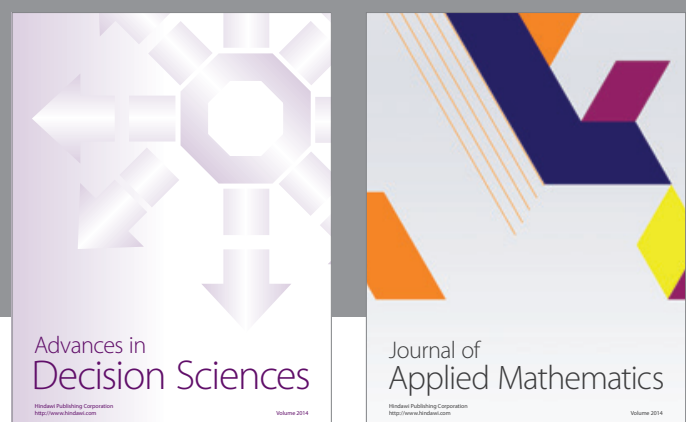

Journal of

Applied Mathematics
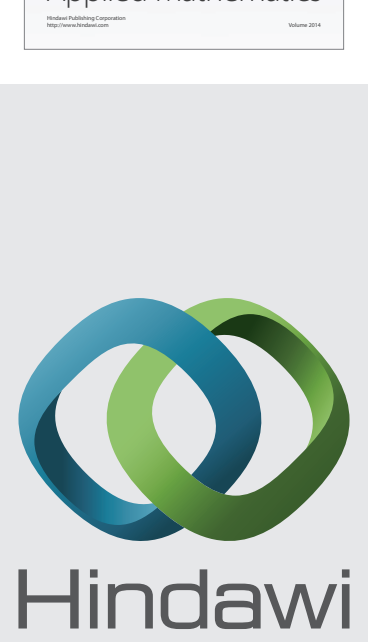

Submit your manuscripts at http://www.hindawi.com
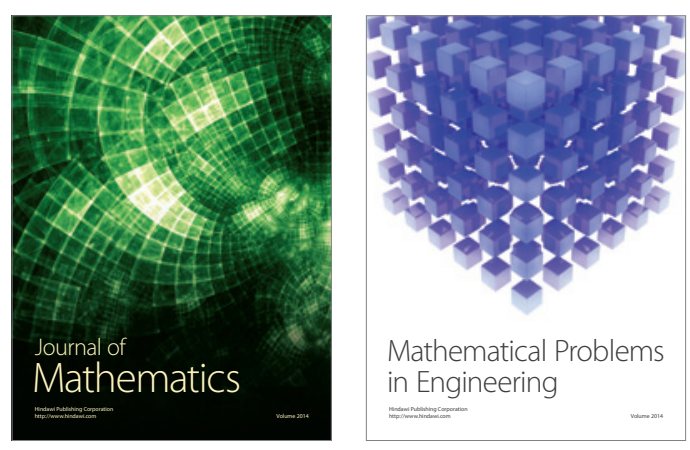

Mathematical Problems in Engineering
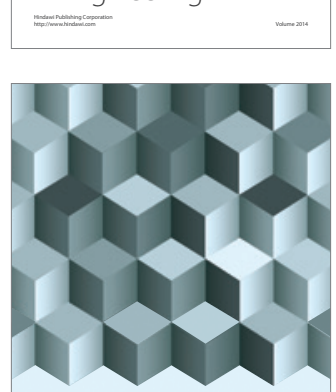

Journal of

Function Spaces
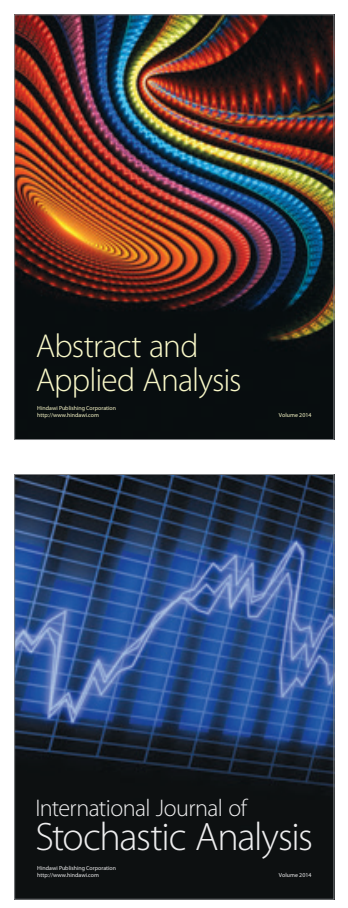

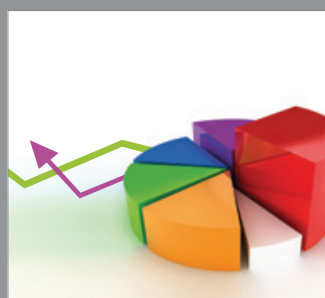

ournal of

Probability and Statistics

Promensencen
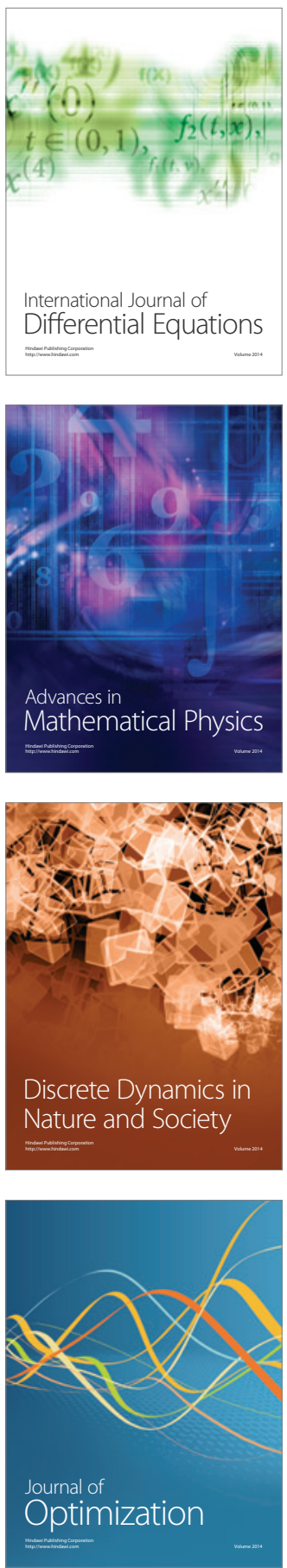